\title{
Sleep-wake pattern of medical students: early versus late class starting time
}

P.F. Lima, A.L.D. Medeiros and J.F. Araujo
Laboratório de Cronobiologia, Departamento de Fisiologia,

Universidade Federal do Rio Grande do Norte, Natal, RN, Brasil

\section{Correspondence}

J.F. Araujo

Laboratório de Cronobiologia

Departamento de Fisiologia, UFRN

Caixa Postal 1506

59078-970 Natal, RN

Brasil

Fax: +55-084-215-3410

E-mail: araujo@cb.ufrn.br

Received November 26, 2001

Accepted July 23, 2002

\begin{abstract}
The sleep-wake cycle of students is characterized by delayed onset, partial sleep deprivation and poor sleep quality. Like other circadian rhythms, the sleep-wake cycle is influenced by endogenous and environmental factors. The aim of the present study was to determine the effects of different class starting times on the sleep-wake pattern of 27 medical students. The data were collected during two medical school semesters having different class starting times. All subjects answered the Portuguese version of the Horne and Östberg Morningness/Eveningness Questionnaire, the Pittsburgh Sleep Quality Index (PSQI) and kept a sleep diary for two weeks during each semester. Better sleep quality (PSQI $=5.3$ vs 3.4), delayed sleep onset (23:59 vs $0: 54 \mathrm{~h}$ ) and longer sleep duration (6 h and $55 \mathrm{~min} v s 7 \mathrm{~h}$ and $25 \mathrm{~min}$ ) were observed with the late schedule. We also found reduced sleep durations during weekdays and extended sleep durations during weekends. This pattern was more pronounced during the semester with the early class schedule, indicating that the students were more sleep deprived when their classes began earlier in the morning. These results require further investigation regarding the temporal organization of our institutions.
\end{abstract}

Human beings, like many other species, organize their activities according to a 24-h cycle. This temporal organization is the result of the interaction of endogenous and environmental factors. Among them, social factors seem to be the most important (1).

The sleep-wake cycle, one of our biological rhythms, is driven by a circadian timing system including the suprachiasmatic nuclei, and is influenced by physiological functions, the light-dark cycle, school and work schedules, leisure and other activities. The chronotype also plays an important role in this cycle. Morning types tend to begin their activities earlier in the morning and evening types tend to delay the initiation of activity.
Key words - Sleep-wake cycle - Class schedule - Sleep quality - Sleep deprivation ...................
The most widely accepted model representing the sleep-wake cycle consists of two components, the circadian and the homeostatic processes. The first depends on the biological circadian timing system and is synchronized by environmental cues and the second depends on the duration of the previous wake or sleep phase (2).

University students are submitted to a lot of pressure due to academic demands. Moreover, the sleep-wake cycle of the students is characterized by insufficient sleep duration, delayed sleep onset and occurrence of napping episodes during the day (3). Sleep deprivation can be harmful to students. A high correlation has been demonstrated between 
sleep duration and performance in some activities, as well as subjective alertness (4). The importance of sleep duration was also shown in our previous study, in which we demonstrated a correlation between sleep deprivation and academic performance in medical students (5).

In the present study we investigated the pattern of the sleep-wake cycle in a natural condition. Our volunteers had two different class schedules during the year and this made it possible for us to compare the early with the late schedule and show the correlation between these schedules, partial sleep deprivation, and sleep quality.

The subjects were medical students from the Universidade Federal do Rio Grande do Norte, Brazil. The data were collected during two semesters. During the first semester, with classes beginning either at 7:00 am (on Tuesdays and Thursdays) or at 8:00 am (on Mondays, Wednesdays and Fridays), 35 students answered our questionnaires. During the second semester, when classes began at 10:00 am every weekday, 31 students participated. For the present study, we used only the data from the volunteers that participated in both the early and the late semester scheduled classes. These students were 13 males and 14 females aged on average $20.2 \pm 2.0$ years.

The participants filled out an identification form with their personal data, class schedules, extracurricular activities and health status. Each volunteer signed a written informed consent form. No monetary compensation was given. The experimental protocol was approved by the Ethics Committee of the Universidade Federal do Rio Grande do Norte. To classify the subjects according to their chronotype, we used the Portuguese version of the Horne and Östberg Morningness/Eveningness Questionnaire (6). The students also answered the Pittsburgh Sleep Quality Index (PSQI) (7), which contains 10 questions related to normal sleep habits. A value above 5 on this test indicates a poor sleep quality. To study the sleep habits, we used the sleep diary. This subjective record is frequently used in sleeprelated research and is highly correlated with polysomnography and Actigraph measures $(8,9)$. All students kept a sleep diary for two consecutive weeks, including three weekends, in which they recorded their bedtimes and rising times. The same protocols were used for both periods of data collection.

To analyze the results of the Horne and Östberg Questionnaire and the PSQI, we constructed a normal distribution curve using the data from the sleep diary. In the analysis we used sleep onset and sleep duration as quantitative variables. The Horne and Östberg Questionnaire, the PSQI and the sleep diary data were compared between semesters using the paired $t$-test.

The values of the Horne and Östberg Questionnaire for each semester were fitted to a normal distribution curve and the difference between semesters was found to be non-significant, with a mean value of 48.5 in the early class schedule and of 46.9 in the late class schedule. In order to study the pattern of the sleep-wake cycle for different class schedules, we used the paired $t$-test to compare bedtime, sleep duration and PSQI scores of the different semesters.

Analysis of the PSQI showed a statistically significant $(\mathrm{P}<0.001)$ improvement of the sleep quality of the students during the late schedule. During the early schedule, $42.3 \%$ of the students had a value above 5 on the test, being therefore classified as having a poor sleep quality. During the late schedule, this decreased to $11.5 \%$ of the students.

The data of the sleep diary showed that mean sleep onset was 23:59 $\mathrm{h}$ and $0: 54 \mathrm{~h}$ for the early and late schedules, respectively. This difference between schedules was statistically significant $(\mathrm{P}=0.004)$. The difference in sleep duration between the two schedules was also statistically significant $(\mathrm{P}<0.001)$, with a mean of $6 \mathrm{~h}$ and $55 \mathrm{~min}$ for the early schedule and $7 \mathrm{~h}$ and $25 \mathrm{~min}$ for the 
late schedule.

We also separated the weekday and weekend data. The sleep onset time and the sleep duration of the weekdays and the weekends are shown in Table 1. During the early class schedule, the weekend delay of sleep onset was about half an hour $(\mathrm{P}=0.02)$ and sleep duration was about $1 \mathrm{~h}$ longer on the weekends $(\mathrm{P}<0.001)$. During the late class schedule, the students went to bed 49 min later on weekends compared to weekdays $(\mathrm{P}<0.001)$ and sleep duration increased by 22 min during the weekends $(\mathrm{P}=0.02)$.

Sleep episodes on the weekdays of the late class schedule began 38 min later than on the weekdays of the early schedule $(\mathrm{P}=$ $0.004)$ and were 40 min longer $(\mathrm{P}<0.001)$. During the weekends, the sleep episodes occurred 56 min later during the late schedule $(\mathrm{P}<0.001)$, but sleep duration remained the same $(P=0.4)$.

The chronotype score of the students did not change during the study. This indicates that chronotype is an endogenous characteristic not influenced by the class schedules.

A relationship has been detected between sleep and measures of health and well-being in college students (10). Although it has been suggested that increasing sleep duration might not directly improve quality of life (11), in our study we found an improvement in the quality of sleep of the students on the late class schedule. During this semester, the students had the opportunity to increase their sleep duration, which probably influenced their sleep quality. However, we did not take into account only sleep duration but also studied the quantitative and qualitative aspects of the sleep-wake cycle, including the differences in the sleep-wake pattern on weekdays and weekends.

The delay of the sleep episodes on the weekends is probably due to both social and endogenous factors. Having no classes the following day, students are free to participate in night activities. Furthermore, humans tend to delay their sleep episode on free days because they have a 25 -h sleepwake cycle (1). Thus, the delayed sleep episodes in the semester during which classes began later can be explained by this endogenous tendency to delay the sleep-wake cycle in addition to the opportunity to wake up and go to bed later (12).

The pattern of reduced sleep length during the weekdays and extended sleep length during the weekends is called restriction/ extension pattern and indicates partial sleep deprivation (Table 1). Having to wake up early during the whole week to go to classes, the students can only recover their sleep during the weekends. During the early class schedule, we observed that $88.9 \%$ of the students showed the restriction/extension pattern. This percentage decreased to $66.7 \%$ during the late class schedule. Since the restriction/extension pattern was more pronounced during the early schedule, we suggest that these students were more sleep deprived during this period, when classes started earlier in the morning. The lack of an increase in the weekend sleep duration of the late schedule compared to the early class schedule is another sign of the existence of partial sleep deprivation during the early class schedule.

Table 1. Sleep variables on weekdays and weekends during the early and late class schedules.

\begin{tabular}{lrr}
\hline Class schedule & \multicolumn{1}{c}{ Weekdays } & \multicolumn{1}{c}{ Weekends } \\
\hline Early schedule & & \\
Sleep onset & $23: 47 \pm 57 \mathrm{~min}$ & $0: 18 \pm 77 \mathrm{~min}$ \\
Sleep offset & $6: 31 \pm 47 \mathrm{~min}$ & $8: 12 \pm 81 \mathrm{~min}$ \\
Sleep duration & $6 \mathrm{~h} 37 \mathrm{~min} \pm 52 \mathrm{~min}$ & $7 \mathrm{~h} 39 \mathrm{~min} \pm 59 \mathrm{~min}$ \\
& & \\
Late schedule & & \\
Sleep onset & $0: 25 \pm 58 \mathrm{~min}$ & $1: 14 \pm 65 \mathrm{~min}$ \\
Sleep offset & $7: 43 \pm 72 \mathrm{~min}$ & $8: 54 \pm 85 \mathrm{~min}$ \\
Sleep duration & $7 \mathrm{~h} 17 \mathrm{~min} \pm 50 \mathrm{~min}$ & $7 \mathrm{~h} 39 \mathrm{~min} \pm 59 \mathrm{~min}$
\end{tabular}

Data are reported as means \pm SD for 27 medical students who participated in both class schedules. Early classes began either at 7:00 am (on Tuesdays and Thursdays) or at 8:00 am (on Mondays, Wednesdays and Fridays) and late classes began at 10:00 am every weekday. Paired $t$-test showed a statistically significant difference between class schedules in sleep onset on weekdays and weekends, and in sleep duration only for the early class schedule. 
Short sleeping time on weekdays combined with an irregular sleep-wake schedule during the week is the most common predictor for daytime sleepiness (13). In a study with 386 adolescents, $38 \%$ of the subjects reported daytime sleepiness (14). Our studies show that our subjects are sleep deprived; the consequences, sleepiness and poor attention during classes, can influence the learning processes.

Sleep deprivation is partly due to class schedules. The morning type students are easily adapted to early morning classes but this is not the case for the evening type students. When classes start later in the morning there is less sleep deprivation and sleep quality is better because both types of students are easily adapted: the evening types can wake up later and the morning types can have other activities before classes if they so wish. Therefore, these considerations should make us question the temporal organization of our institutions and require a deeper analysis of the influence of class schedules on the academic performance of the students.

Performance of tasks during times of day when psychomotor capabilities are suboptimal is associated with many serious consequences. Studies conducted with professionals submitted to shift work have revealed that a high percentage of errors during work is due to partial sleep deprivation. The various effects of sleep deprivation include neurophysiological alterations like decreased alertness, psychological alterations like mood variations, and direct effects on performance (15). For example, nurses on a repetitive shift work schedule are two- to three-fold more likely to wrongly care for patients than their daytime colleagues (16).

Our subjects were medical students who, like most medical students, were submitted to a demanding curricular schedule in addition to stress due to the constant contact with human suffering and death. The large number of classes and the need to devote much time to studying contributed to the sleep deprivation observed in this group of students. In contrast, during the late class schedule, students had longer sleep duration and better sleep quality. Furthermore, there was a decrease in the percentage of students who showed the restriction/extension pattern, suggesting that they were less sleep deprived during this period. Thus, the present study showed that the time of class initiation in the morning can influence the students' sleepwake pattern and, based on our previous work, we believe that the sleep-wake pattern can affect academic performance.

\section{References}

1. Aschoff J (1965). Circadian rhythms in man. Science, 148: 1427-1432.

2. Borbély AA (1982). Two process model of sleep regulation. Human Neurobiology, 1: 195-204

3. Jean-Louis G, Von Gizycky H, Zizi F \& Nunes J (1998). Mood states and sleepiness in college students: influences of age, sex, habitual sleep and substance use. Perceptual and Motor Skills, 87: 507512.

4. Jewett ME, Dijk DJ, Kronauer RE \& Dinges DF (1999). Dose-response relationship between sleep duration and human psychomotor vigilance and subjective alertness. Sleep, 22: 171-179.

5. Medeiros AL, Mendes DBF, Lima PF \&
Araujo JF (2001). The relationships between sleep-wake cycle and academic performance in medical students. Biological Rhythm Research, 32: 263-270.

6. Horne JA \& Östberg O (1976). A selfquestionnaire to determine morningnesseveningness in human circadian rhythms. International Journal of Chronobiology, 4 97-110

7. Buysse DJ, Reynolds CF, Monk TH, Berman SR \& Kupfer DJ (1989). The Pittsburg sleep quality index: a new instrument for psychiatric practice and research. Psychiatry Research, 28: 193-213.

8. Lockley SW, Skene DJ \& Arendt J (1999) Comparison between subjective and actigraphic measurement of sleep and sleep rhythms. Journal of Sleep Research, 8: $175-183$

9. Usui A, Ishizuka $Y$, Obinata I, Okado T, Fukuzawa H \& Kanba S (1999). Validity of sleep log compared with actigraphic sleep-wake state II. Psychiatry and Clinical Neurosciences, 53: 183-184.

10. Pilcher J \& Otto ES (1998). The relationships between sleep and measures of health and well-being in college students: a repeated measures approach. Behavioral Medicine, 23: 170-177.

11. Jean-Louis G, Kripke DF \& Ancoli-Israel S (2000). Sleep and quality of well-being. Sleep, 23: 1115-1121.

12. Valdez P, Ramírez C \& Garcia A (1996). Delaying and extending sleep during 
weekends: sleep recovery or circadian effect? Chronobiology International, 13: 191-198.

13. Saarenpää-Heikkilä OA, Rintahaka PJ, Laippala PJ \& Koivikko MJ (2000). Subjective daytime sleepiness and related predictors in Finnish schoolchildren. Sleep and Hypnosis, 2: 139-146.
14. Mantz J, Muzet A \& Winter AS (2000). The characteristics of sleep-wake rhythm in adolescents aged 15-20 years: a survey made at school during ten consecutive days. Archives de Pediatrie, 7: 256-262.

15. Gaspar S, Moreno C \& Menna-Barreto L (1998). Os plantões médicos, o sono e a ritmicidade biológica. Revista da Associa- ção Médica Brasileira, 44: 239-245.

16. Gold DR, Rogacz S, Bock N, Tosteson TD, Baum TM, Speizer FE \& Czeisler CA (1992). Rotating shift work, sleep, and accidents related to sleepiness in hospital nurses. American Journal of Public Health, 82: 1011-1014. 